Forum 2018 · 33:6

https://doi.org/10.1007/s12312-017-0378-8

Online publiziert: 9. Januar 2018

(c) Springer Medizin Verlag $\mathrm{GmbH}$, ein Teil von Springer Nature 2018

\title{
Der DKK 2018 - neue Perspektiven in der Onkologie
}

Häufig kommen sie aber gar nicht zu den Aufgaben, für die sie speziell weitergebildet ist; aufgrund des Pflegenotstands wird ihre Arbeitskraft vielmehr dafür benötigt, um die Grundpflege auf der Station aufrechtzuerhalten. Was sich an der Situation in der onkologischen Pflege ändern lässt, darüber werden wir auf dem Kongress diskutieren.

Ein anderes Thema von hoher Brisanz wird auf dem Kongress hoffentlich auch zur Sprache kommen: Im Rahmen des Pharmadialogs kam man zum Schluss, dass die Beschlüsse der frühen Nutzenbewertung durch den G-BA künftig in die ärztliche Praxissoftware integriert werden sollen. Im Vordergrund dieser Überlegung stehen wirtschaftliche Aspekte - Ärzte sollen sich bei ihren Verschreibungen an der Nutzenbewertung orientieren. Denn diese ist ein Instrument zur ökonomischen Steuerung - auf der Basis des vom G-BA festgestellten Zusatznutzens im Vergleich zur Standardtherapie wird der Preis eines neuen Medikaments festgelegt. Die Nutzenbewertung unterscheidet sich aber grundlegend von der wissenschaftlichen Interpretation der Evidenzlage. Insbesondere die durch den G-BA definierten Patientensubgruppen sind in den meisten Studien nicht prospektiv in der Studienplanung und Fallzahlkalkulation berücksichtigt worden. Daher weicht auch die Definition von Patientensubgruppen in der frühen Nutzenbewertung des G-BA häufig von den klinisch relevanten Subgruppen in medizinischen Leitlinien ab.

\section{》) Wer legt fest, was der allge- meine Stand des medizinischen Wissens ist?}

Mittlerweile liegt ein aktuelles Gutachten vor, das die Beschlüsse der frühen Nutzenbewertung bei Krebsmedikamenten mit dem allgemein anerkannten Standard medizinischen Wissen vergleicht, wie er zum
Beispiel von der DGHO für die Plattform Onkopedia formuliert wird. Es zeigt einen partiellen oder kompletten Widerspruch bei $60 \%$ der untersuchten Patientengruppen über alle Tumorarten hinweg [1]. Diskrepanzen finden sich auch, wenn man die S3-Leitinien des Leitlinienprogramms Onkologie zugrunde legt: Die aktuelle S3Leitlinie Lungenkarzinom kommt zum Beispiel zum Schluss, dass Crizotinib bei Patienten mit einem nicht-kleinzelligen Lungenkarzinom (NSCLC) und ROS1Fusionsgenen in der Erstlinientherapie empfohlen werden soll, und zwar mit einem hohen Evidenzgrad. In der frühen Nutzenbewertung gilt der Zusatznutzen für Crizotinib in dieser Patientengruppe als nicht belegt.

Wer legt fest, was der allgemeine Stand des medizinischen Wissens ist? Per Definition ist das eine ärztliche Aufgabe, keine der Kassen oder Selbstverwaltung. Wieso bildet man nicht den allgemeinen Stand des medizinischen Wissens in der PraxisSoftware ab, etwa durch das Einstellen medizinischer Leitlinien? Für eine Versorgung, die sich am optimalen Patientennutzen orientiert, wäre das sicher hilfreich. Ich freue mich auf Ihre Meinung dazu und auf viele anregende Diskussionen auf dem DKK 2018.

Ihr

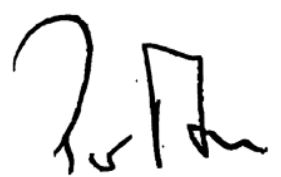

Peter Albers

Präsident der Deutschen Krebsgesellschaft

\section{Literatur}

1. https://www.vfa.de/de/download-manager/_ analyse-widersprueche-leitlinienempfehlungengba.pdf. Zugegriffen: 01. Dezember 2017 